\title{
Quantitative AES, XPS and RBS determination of intergranular bismuth coverage in copper bicrystals at $500{ }^{\circ} \mathbf{C}$
}

\author{
V. Laporte, ${ }^{1,2 *}$ P. Berger ${ }^{3}$ and K. Wolski ${ }^{1}$ \\ ${ }^{1}$ Centre Science des Matériaux et des Structures, Ecole Nationale Supérieure des Mines de Saint-Etienne, CNRS PECM UMR 5146, 158, cours Fauriel, \\ F-42023 Saint-Etienne cedex 02, France \\ 2 Ecole Polytechnique Fédérale de Lausanne (EPFL), Laboratoire de Métallurgie Mécanique, Station 12, CH-1015 Lausanne, Switzerland \\ ${ }^{3}$ Laboratoire Pierre SÜE (CEA/CNRS), UMR9956 CEA/SACLAY, F-91191 Gif Sur Yvette Cedex, France
}

Received 16 December 2004; Revised 30 June 2005; Accepted 13 July 2005

\begin{abstract}
We report on a comparative measurement of intergranular bismuth coverage on a copper substrate using Auger electron spectroscopy (AES), x-ray photoelectron spectroscopy (XPS) and Rutherford backscattering spectroscopy (RBS). Bicrystalline copper samples were put in presence of bismuth vapour at $500^{\circ} \mathrm{C}$ (consequently embrittled by the grain-boundary penetration of $\mathrm{Bi}$ atoms), water-quenched and subsequently fractured at room temperature. Each fracture surface was analysed by AES, XPS and RBS with the help of quantitative procedures developed for each of the three techniques. All possible sources of discrepancy were carefully examined. The combined quantitative approaches have led to excellent agreement. Such a good agreement constitutes a necessary condition to begin a critical discussion on the mechanisms potentially involved in the liquid metal embrittlement (LME) phenomenon. Copyright (C) 2005 John Wiley \& Sons, Ltd.
\end{abstract}

KEYWORDS: liquid metal embrittlement; intergranular penetration; AES; XPS; RBS

\section{INTRODUCTION}

Intergranular brittleness of metallic materials is known to be the result of either impurity segregation from the bulk material or intergranular diffusion of embrittling species from an aggressive external environment. When such an environment is constituted by liquid lead-bismuth used in spallation target of hybrid systems, ${ }^{1-3}$ it can result notably in the liquid metal embrittlement (LME) phenomenon.

In spite of a number of proposed mechanisms reviewed in many articles, ${ }^{4,5}$ there is still a lack of understanding, especially, in the prediction of LME. In order to determine the mechanism controlling this phenomenon, the absolute amount of heavy metal in the grain boundaries must be known with precision. In fact, if this quantity is in the range one or two monolayers, intergranular diffusion, until equilibrium segregation, could be responsible for the observed embrittlement. Otherwise (thickness $\gg 2$ monolayers), it would appear inevitable to introduce a grainboundary wetting formalism, where grain boundaries can be replaced by liquid phases after being penetrated by foreign atoms. Hence, the precise determination of the thickness can actually give valuable information about elementary mechanisms involved in the embrittlement.

*Correspondence to: V. Laporte, Ecole Polytechnique Fédérale de Lausanne (EPFL), Laboratoire de Métallurgie Mécanique, Station 12, CH-1015 Lausanne, Switzerland. E-mail: vincent.laporte@epfl.ch
This is the reason why we decided to use three independent methods: Auger electron spectroscopy (AES), x-ray photoelectron spectroscopy (XPS) and Rutherford backscattering spectroscopy (RBS), all of them known for their ability to produce quantitative results. We have chosen to study this phenomenon using a model system known for its LME predisposition: ${ }^{6}$ solid copper in contact with liquid bismuth. One of the main interests of this system is that copper grain boundaries remain brittle at room temperature if they have been previously exposed to bismuth, ${ }^{7}$ and this brittleness allows first AES and XPS analyses after in situ fractures, and second RBS analyses, all of them on the same specimen and the same fracture surface.

This kind of study has been already done for another similar system: solid nickel in contact with liquid bismuth, where bismuth has an even stronger intergranular embrittling effect. The authors ${ }^{8,9}$ established that polycrystalline nickel was embrittled by a nanometer-thick intergranular film of a bismuth-rich alloy after heat treatment at $700{ }^{\circ} \mathrm{C}$ where nickel, either directly or through its vapour was in contact with oversaturated bismuth. This work also suggests the use of vapour contact instead of direct contact to observe bismuth embrittlement of copper.

The purpose of the present paper is to quantify the amount of bismuth present in copper grain boundaries using independent AES, XPS and RBS measurements made on the same fracture surfaces of bicrystalline copper specimens embrittled by contact with liquid bismuth. These results 
will be shortly discussed with respect to potentially efficient mechanisms of LME.

\section{EXPERIMENTAL}

The copper bicrystal used in this study had a $50^{\circ}\langle 100\rangle$ tilt grain boundary. It was prepared by melting and controlled solidification in a horizontal furnace (using a bicrystalline seed on an alumina mould) and cut by spark erosion to get parallelepipeds $(1.5 \times 1.5 \times 20 \mathrm{~mm})$ with the grain boundary at approximately half the distance along the length. It was then chemically polished in order to remove residual stresses introduced by spark erosion (thus avoiding any recrystallisation during the subsequent heat treatment).

Each copper specimen and a few pieces of pure solid bismuth were placed in a silica tube sealed under argon, in such a way that no direct contact between the two metals was possible during the heat treatment (Fig. 1). This procedure had a twofold advantage: (i) it avoided copper dissolution into liquid bismuth, the rate of which can reach several tens of microns per hour ${ }^{10}$ and (ii) it limited the quantity of bismuth introduced in the AES-XPS main chamber, thereby limiting possible bismuth evaporation problems during external heatmaintenance operations.

All heat treatments were performed at $500^{\circ} \mathrm{C}\left(>271^{\circ} \mathrm{C}\right.$, which is the melting point of bismuth) and two time periods were selected in order to get a complete and homogeneous penetration of a unique grain boundary: $48 \mathrm{~h}$ (samples S0481 and S048-2) and $137 \mathrm{~h}$ (samples S137-1 and S137-2). AES analyses were performed for all specimens, XPS analyses were achieved only for S048-1 and S048-2 and RBS analyses for S048-1 and S137-1.

At $500{ }^{\circ} \mathrm{C}$, the total pressure of bismuth vapour is about $4 \times 10^{-8}$ bar, and this value allows the deposition of several nanometers of bismuth on the external surfaces of the bicrystal specimen as revealed by glow discharge optical spectroscopy. During the heat treatment, this liquid layer can act as a source for the bismuth grain boundary penetration into copper. This procedure has been suggested by the previous work of Fraczkiewicz at the Ecole des Mines de Saint-Etienne on the study of the intergranular segregation of bismuth in copper bicristals, ${ }^{11,12}$ also applied by Marié of the same laboratory to study the intergranular penetration of bismuth into nickel bicristals and polycrystals ${ }^{8,13}$ and, probably, independently developed by Chang in his recent thesis on the intergranular segregation of bismuth in copper polycrystals. ${ }^{14}$ Such a procedure was thus shown to be

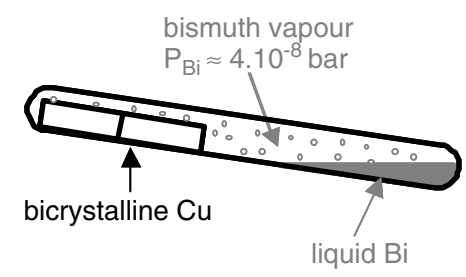

Figure 1. Experimental procedure: bicrystalline $\mathrm{Cu}$ and pure $\mathrm{Bi}$ are placed in a silica tube sealed under argon so that no direct contact is possible during heat treatment in the furnace at $500^{\circ} \mathrm{C}$. equivalent to the direct contact between copper and bismuthrich $\mathrm{Bi}-\mathrm{Cu}$ alloy. ${ }^{15}$ After the heat treatment, specimens were water-quenched in a few seconds.

In order to reveal the grain boundary composition, the specimens were broken by bending (for S048-1 and S0482) or by tensile test (for S137-1 and S137-2) within the main chamber of the AES-XPS spectrometer. The fracture surfaces were always intergranular and completely brittle, leading to homogeneously flat surfaces. Only one side of each specimen could be analysed after in situ fracture by AES and XPS (Fig. 2), but it is worth noticing that both were analysed by RBS in order to confirm the assumption of equirepartition of bismuth on both sides.

A vacuum of less than $5 \times 10^{-10}$ mbar in the main chamber together with the low reactivity of fracture surfaces allowed an 8-h long series of AES and XPS measurements without any noticeable carbon or oxygen contamination. RBS analyses were performed six months later in the Pierre Süe Laboratory of the French Atomic Energy Commission (CEA). A detailed description of the quantification procedures used for each technique is presented here and, in the following, we will use $\tau_{A E S}, \tau_{X P S}$ and $\tau_{R B S}$ to refer to the amount of bismuth (expressed as fraction of monolayer) determined using the corresponding techniques. These values correspond to the amount of bismuth on one fracture surface; therefore, the total amount of bismuth in the grain boundary is the double, i.e. $2 \times \tau$.

\section{AES quantification}

AES analyses were performed under a $5-\mathrm{keV}$ primary electron beam incident at an angle of $\varphi=50^{\circ}$ with respect to the surface normal. AES spectra were recorded with a Cameca Mac III analyser. The emitted electrons were collected at a resolution of $1 \mathrm{eV}$ with a step of $0.25 \mathrm{eV}$ and at an angle of $\theta=20^{\circ}$ with respect to the surface normal. The AES-analysed areas were either about $50 \mu \mathrm{m}^{2}$ spots or several hundreds of $\mu \mathrm{m}^{2}$ scans, made on different places of the fracture surface in order to evaluate the homogeneity of the bismuth coverage.

\section{AES intensities expression}

Assuming that bismuth is present as a fraction of monolayer $\tau_{A E S}$ and using a discrete summation (monolayer by monolayer), the AES bismuth and copper intensities can be expressed as:

$$
I_{B i}^{A E S}=\tau_{A E S} K_{A E S} \beta_{B i} n_{B i} R_{B i}^{C u}
$$

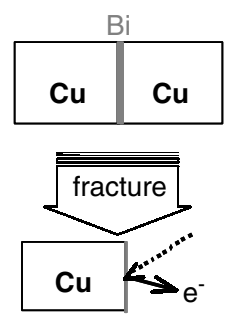

Figure 2. In situ fracture of the embrittled bicrystals: coupled XPS-AES measurements could be performed on only one of the sides of the fracture surface. 
and

$$
\begin{aligned}
I_{C u}^{A E S} & =K_{A E S} \beta_{C u} n_{C u} R_{C u}^{C u}\left[\tau_{A E S} k_{p}^{B i} k_{C u}^{B i}+\left(1-\tau_{A E S}\right)\right] \sum_{i=0}^{+\infty}\left(k_{p}^{C u} k_{C u}^{C u}\right)^{i} \\
& =K_{A E S} \beta_{C u} n_{C u} R_{C u}^{C u}\left[\frac{\tau_{A E S} k_{p}^{B i} k_{C u}^{B i}+\left(1-\tau_{A E S}\right)}{1-k_{p}^{C u} k_{C u}^{C u}}\right]
\end{aligned}
$$

with:

$K_{A E S}$ depending on instrumental factors and analysis conditions $\left(K_{A E S}\right.$ is the same for all elements during the analysis of a given specimen),

$\beta_{A}$ depending on the chosen auger transition for the element $\mathrm{A}$

$n_{A}$ the numbers of $\mathrm{A}$ atoms per unit area,

$R_{A}^{M} \quad$ the backscattering factor for a chosen transition of the element A through the matrix $\mathrm{M}$,

$k_{p}^{M}=\exp \left(\frac{-d_{M}}{\lambda_{p}^{M} \cos \varphi}\right)$ the attenuation factor of primary electrons through the matrix $\mathrm{M}$,

$k_{A}^{M}=\exp \left(\frac{-d_{M}}{\lambda_{A}^{M} \cos \theta}\right)$ the attenuation factor of an electron of $A$ in the matrix $M$,

$d_{M}$ the monolayer thickness of the matrix $\mathrm{M}$ and

$\lambda_{A}^{M}$ the inelastic mean free path for an electron of $\mathrm{A}$ in the matrix $\mathrm{M}\left(\lambda_{p}^{M}\right.$ meaning the inelastic mean free path of the primary electrons through the matrix $\mathrm{M}$ ) [the choice of this parameter instead of the effective attenuation length is addressed in the discussion].

Difficulties in AES quantifications lie essentially in $\beta$ determination that involves the evaluation of the ionisation cross-section, the deexcitation probability and the analyser transmission function. Pure elements (both copper and bismuth) were therefore used to evaluate the ratio $\frac{\beta_{B i}}{\beta_{C i}}$. For pure elements analysed under the same experimental conditions (electron beam and geometrical considerations), the following equations can be written:

$$
\begin{aligned}
I_{B i}^{0} & =K_{A}^{0} \beta_{B i} n_{B i} R_{B i}^{B i} \sum_{i=0}^{+\infty}\left(k_{p}^{B i} k_{B i}^{B i}\right)^{i} \\
& =\frac{K_{A}^{0} \beta_{B i} n_{B i} R_{B i}^{B i}}{1-k_{p}^{B i} k_{B i}^{B i}}
\end{aligned}
$$

and

$$
\begin{aligned}
I_{C u}^{0} & =K_{A}^{0} \beta_{C u} n_{C u} R_{C u}^{C u} \sum_{i=0}^{+\infty}\left(k_{p}^{C u} k_{C u}^{C u}\right)^{i} \\
& =\frac{K_{A}^{0} \beta_{C u} n_{C u} R_{C u}^{C u}}{1-k_{p}^{C u} k_{C u}^{C u}}
\end{aligned}
$$

which leads to:

$$
\frac{\beta_{B i}}{\beta_{C u}}=\frac{I_{B i}^{0} n_{C u} R_{C u}^{C u}}{I_{C u}^{0} n_{B i} R_{B i}^{B i}} \frac{1-k_{p}^{B i} k_{B i}^{B i}}{1-k_{p}^{C u} k_{C u}^{C u}}
$$

and thus:

$$
\frac{I_{B i}^{A E S}}{I_{C u}^{A E S}}=\frac{I_{B i}^{0}}{I_{C u}^{0}} \times \frac{R_{B i}^{C u}}{R_{B i}^{B i}} \times \frac{1-k_{p}^{B i} k_{B i}^{B i}}{\frac{\left(1-\tau_{A E S}\right)}{\tau_{A E S}}+k_{p}^{B i} k_{C u}^{B i}}
$$

It is worth noticing here that the same relationship can be obtained using a continuous summation (see appendix for details).

\section{AES parameters evaluation}

The backscattering factors $R$ were obtained using the empirical formula: ${ }^{16}$

$R_{A}^{M}=1+\left(2.34-2.10 Z_{M}^{0.14}\right) \times\left(\frac{E_{p}}{E_{L}^{A}}\right)^{-0.35}+\left(2.58 Z_{M}^{0.14}-2.98\right)$

With $Z_{C u}=29, Z_{B i}=83, E_{p}=5000 \mathrm{eV}$ and $E_{L}=161.9 \mathrm{eV}$ (for the bismuth $\mathrm{N}_{6} \mathrm{O}_{45} \mathrm{O}_{45}$ transition at $101 \mathrm{eV}$ ), $R_{B i}^{C u} \approx 1.85$, $R_{B i}^{B i} \approx 2.34$ and finally $\frac{R_{B i}^{C u}}{R_{B i}^{B i}} \approx 0.8$.

The bismuth monolayer thickness $d_{B i}$ was evaluated as the cubic root of the atomic volume:

$$
d_{B i} \approx \sqrt[3]{\frac{M_{B i}}{N_{a v o} \rho_{B i}}}
$$

With $M_{B i} \approx 208.98 \mathrm{~g} \mathrm{~mol}^{-1}$ the molar mass, $N_{a v o}$, the number of atoms per mole and $\rho_{B i} \approx 9.8 \mathrm{~g} \mathrm{~cm}^{-3}$ the density, that leads to $d_{B i} \approx 0.329 \mathrm{~nm}$.

The inelastic mean free paths (IMFP) $\lambda_{A}^{M}$ were determined using the semiempirical formula: ${ }^{17-19}$

$$
\lambda_{A}^{M}=\frac{E_{c}^{A}}{\left(E_{p}^{M}\right)^{2} \times\left[B_{M} \times \ln \left(\gamma_{M} E_{c}^{A}\right)-\frac{C}{E_{c}^{A}}+\frac{D}{\left(E_{c}^{A}\right)^{2}}\right]}
$$

with $\lambda_{A}^{M}$ expressed in angstroms and $E_{c}$ (kinetic energy) in electronvolts and:

$$
E_{p}^{M}=28.8 \times \sqrt{\frac{N_{v}^{M} \times \rho_{M}}{\mathrm{M}_{M}}}
$$

( $N_{V}$ : number of valence electrons, $\rho$ : density and $M$ : molar mass)

$$
\begin{aligned}
B_{M} & =-0.1+\frac{0.944}{E_{p}^{M}}+0.069\left(\rho_{M}\right)^{0.1} \\
\gamma_{M} & =0.191\left(\rho_{M}\right)^{-0.5} \\
C_{M} & =1.97-0.91 \times U_{M} \\
D_{M} & =53.4-20.8 \times U_{M} \\
U_{M} & =\frac{\left(E_{p}^{M}\right)^{2}}{829.4}
\end{aligned}
$$

The transitions used in the analyses were the $\mathrm{Cu} \mathrm{M} \mathrm{M}_{23} \mathrm{VV}$ (kinetic energy: $59 \mathrm{eV}$ ) and the $\mathrm{Bi}_{6} \mathrm{O}_{45} \mathrm{O}_{45}$ (kinetic energy: $101 \mathrm{eV})$. The IMFP were then calculated:

$$
\begin{aligned}
\lambda_{p}^{B i} & \approx 8.274 \mathrm{~nm}(\approx 25.2 \text { monolayers of bismuth }) \\
\lambda_{B i}^{B i} & \approx 0.602 \mathrm{~nm}(\approx 1.8 \text { monolayers of bismuth }) \\
\lambda_{C u}^{B i} & \approx 0.564 \mathrm{~nm}(\approx 1.7 \text { monolayers of bismuth })
\end{aligned}
$$

Then, Eqn (6) becomes:

$$
\tau_{A E S}=\frac{\frac{I_{B i}^{A E S}}{I_{C u}^{A E S}}}{0.49 \times \frac{I_{B i}^{A E S}}{I_{C u}^{A E S}}+0.38 \times \frac{I_{B i}^{0}}{I_{C u}^{0}}}
$$




\section{AES results}

Experimental intensities were determined using peak-topeak heights evaluated after differentiation of the smoothed direct spectra (Fig. 3). With such a procedure, the experimental standard intensity ratio $\frac{I_{B i}^{0}}{I_{\mathrm{C} u}^{0}}$ measured on pure elements was found to be about $1: \frac{I_{B i}^{0}}{I_{C u}^{0}} \approx 1 \pm 0.1$. The fraction of bismuth monolayer $\tau_{A E S}$ present on the fracture surface is then related to the experimental bismuth-to-copper intensity ratio with the following equation:

$$
\tau_{A E S} \approx \frac{\frac{I_{B i}^{A E S}}{I_{C u}^{A E S}}}{0.49 \times \frac{I_{B i}^{A E S}}{I_{C u}^{A E S}}+0.38}
$$

Experimental bismuth-to-copper AES intensity ratios and corresponding fractions of the bismuth monolayer are presented in Table 1.

In addition to the discrepancies introduced by the dispersion of experimental results, those due to the quantification parameter evaluation are to be discussed: namely, the IMFP evaluation, the experimental standard intensity ratio $\frac{I_{B i}^{0}}{I_{C u}^{0}}$ evaluation and the primary electron beam attenuation can modify Eqn (17). First, the IMFP can be evaluated using another empirical equation ${ }^{20}$ different from that proposed in the previous section:

$$
\lambda_{A}^{M}=538\left(E_{c}^{A}\right)^{-2}+0.41 \sqrt{d_{M} E_{c}^{A}}
$$

With such a relationship, the IMFP become:

$\lambda_{p}^{B i} \approx 5.471 \mathrm{~nm}(\approx 16.6$ monolayers of bismuth $)$

$\lambda_{B i}^{B i} \approx 0.795 \mathrm{~nm}(\approx 2.4$ monolayers of bismuth $)$

$\lambda_{\mathrm{Cu}}^{B i} \approx 0.645 \mathrm{~nm}(\approx 2.0$ monolayers of bismuth $)$

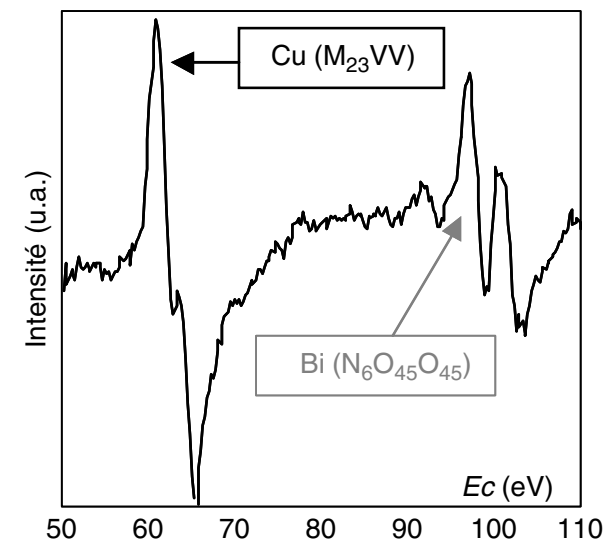

Figure 3. Example of a differential AES spectrum obtained for S048-1 (in situ fractured Cu 48 h-specimen).
As a result, Eqn (17) changes into Eqn (19) and produces a maximum deviation from our first $\tau_{A E S}$ calculation of less than +0.08 (Fig. 4).

$$
\tau_{A E S} \approx \frac{\frac{I_{B i}^{A E S}}{I_{C u}^{A E S}}}{0.48 \times \frac{I_{B i}^{A E S}}{I_{C u}^{A E S}}+0.33}
$$

Second, the discrepancy of the experimental $\frac{I_{B i}^{0}}{I_{C}^{0}}$ ratio induces a maximum deviation from our $\tau_{A E S}$ calculation of less than \pm 0.05 (Fig. 5). Lastly, the primary electron beam attenuation is usually neglected in AES quantification, but it is kept under consideration in our study. The first reason is that the intensity expressions are more rigorous and general. The second lies in the numerical values obtained for this attenuation, which can reach significant level:

$\exp \left(\frac{-d_{B i}}{\lambda_{p}^{B i} \cos \varphi}\right) \approx \begin{gathered}0.94 \text { if we use the first semiempirical } \\ \text { expression presented for the inelastic }\end{gathered}$ mean free path ${ }^{17,18}$ and

$\exp \left(\frac{-d_{B i}}{\lambda_{p}^{B i} \cos \varphi}\right) \approx 0.91$ if we use the second one..$^{20}$

On another hand, the other parameters do not introduce any significant variation in our results. For example, even if several relations exist for the backscattering factor evaluation, ${ }^{16,21}$ the evaluation of the ratio $\frac{R_{B i}^{C u}}{R_{B j}^{B i}}$ is not significantly altered by the use of these different relations.

Taking into account the above mentioned uncertainties allows us to conclude that bismuth monolayer fraction given by AES measurements is $\tau_{A E S}=82 \% \pm 18 \%$.

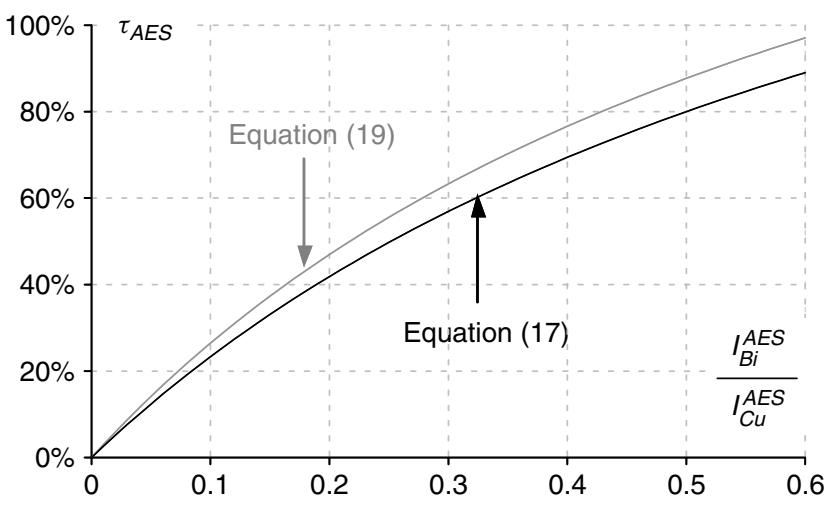

Figure 4. Graphical evolution of $\tau_{A E S}$ as a function of $\frac{l_{B i}^{A E S}}{l_{C E S}^{A E S}}$ depending on the empirical equation chosen for the inelastic mean free path evaluation: black curve is obtained with Eqn (17) and grey curve with Eqn (19).

Table 1. Experimental bismuth-to-copper AES intensity ratios (obtained using peak-to-peak evaluation after differentiation of the smoothed direct AES spectra) and their corresponding fractions of bismuth monolayer $\tau_{A E S}$ obtained using Eqn (17): indicated discrepancies are only those introduced by the dispersion of results

\begin{tabular}{lcccc}
\hline & $S 048-1$ & $S 048-2$ & $S 137-1$ & $S 137-2$ \\
\hline$I_{B i}^{A E S}$ & $0.51 \pm 0.02(10$ analyses $)$ & $0.50 \pm 0.02$ (4 analyses) & $0.60 \pm 0.02$ (5 analyses) & $0.43 \pm 0.03(4$ analyses $)$ \\
$I_{C u}^{A E S}$ & $0.81 \pm 0.04$ & $0.80 \pm 0.06$ & $0.89 \pm 0.06$ & $0.73 \pm 0.08$ \\
$\tau_{A E S}$ & & & 0.06 \\
\hline
\end{tabular}




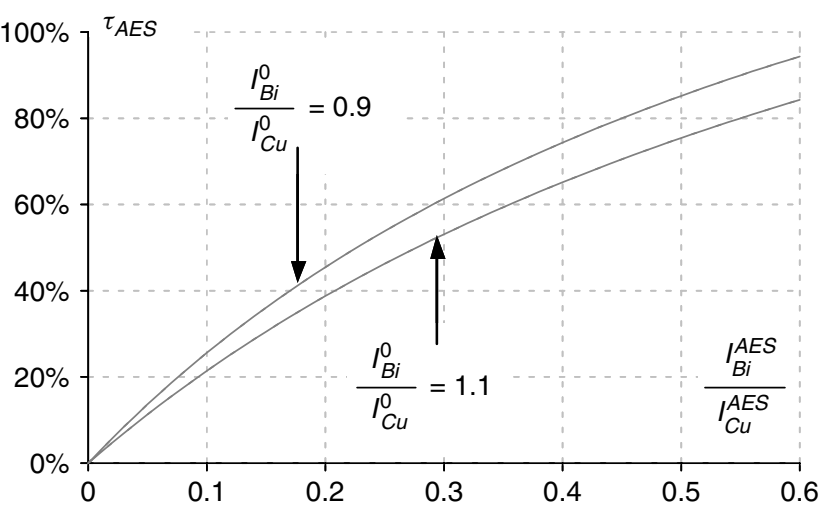

Figure 5. Graphical evolution of $\tau_{A E S}$ as a function of $\frac{l_{B i}^{A E S}}{l_{C i}^{A E S}}$ representing the discrepancy due to the experimental evaluation of $\frac{l_{B i}^{0}}{l_{C u}^{0}}$ ratio: both curves are obtained using Eqn (16).

\section{XPS quantification}

After the in situ fracture of the specimens, one each of S048-1 and S048-2 fracture surfaces were excited with $\mathrm{Mg} \mathrm{K} \alpha$ Xrays incident at an angle of $72^{\circ}$ with respect to the surface normal. X-ray-excited photoelectron spectra were recorded with a Cameca Mac III analyser. The emitted electrons were collected with a 1-eV energy resolution at an angle of $\theta=20^{\circ}$ with respect to the surface normal. Due to the high spot size of photons from the twin anode source, XPS spot size is greater than the in situ opened fracture surface of $1.5 \times 1.5 \mathrm{~mm}^{2}$. A specific procedure was hence used on both S048-1 and S048-2 before in situ fracture, consisting of gold deposition on their external surfaces in order to suppress any possible contribution of the bismuth-covered lateral surfaces after in situ fracture.

\section{XPS discrete summation}

Assuming again that bismuth is present on copper as a homogeneous fraction of monolayer $\tau_{X P S}$ and using a discrete summation, the XPS bismuth and copper intensities can be expressed as:

$$
I_{B i}^{X P S}=\tau_{X P S} K_{X P S} n_{B i} \sigma_{B i} T_{B i}
$$

and

$$
\begin{aligned}
I_{C u}^{X P S} & =K_{X P S} n_{C u} \sigma_{C u} T_{C u}\left[\left(1-\tau_{X P S}\right)+\tau_{X P S} k_{C u}^{B i}\right] \sum_{i=0}^{+\infty}\left(k_{C u}^{C u}\right)^{i} \\
& =K_{X P S} n_{C u} \sigma_{C u} T_{C u} \frac{\left[\left(1-\tau_{X P S}\right)+\tau_{X P S} k_{C u}^{B i}\right]}{1-k_{C u}^{C u}}
\end{aligned}
$$

with:

$K_{X P S}$ depending on instrumental factors and analysis conditions ( $K_{X P S}$ is the same for all elements during the analysis of a given specimen),

$\sigma_{A}$ the photoionisation cross-section,

$T_{A}$ the transmission function,

$d_{B i}$ the bismuth monolayer thickness and

$\lambda_{A}^{M}$ the inelastic mean free path for an electron of $\mathrm{A}$ in the matrix M.
These expressions lead to:

$$
\tau_{X P S}=\frac{\frac{I_{B i}^{X P S}}{I_{C u}^{X P S}}}{\frac{n_{B i} \sigma_{B i} T_{B i}}{n_{C u} \sigma_{C u} T_{C u}}\left[1-k_{C u}^{C u}\right]+\frac{I_{B i}^{X P S}}{I_{C u}^{X P S}}\left[1-k_{C u}^{B i}\right]}
$$

and, with the chosen transitions $\left(\mathrm{Cu}-2 \mathrm{p}^{3 / 2}\right.$ single peak with a kinetic energy of $319.6 \mathrm{eV}$ and $\mathrm{Bi}-4 \mathrm{f}^{5 / 2}$ and $\mathrm{Bi}-4 \mathrm{f}^{7 / 2}$ peaks with the respective kinetic energies of 1089.6 and $1094.6 \mathrm{eV}$ ), to:

$$
\tau_{X P S}=\frac{\frac{I_{B i}^{X P S}}{I_{C u}^{X P S}}}{\frac{n_{B i}\left[\sigma_{B i-4 f^{7 / 2}}+\sigma_{B i-4 f^{5 / 2}}\right] T_{B i}}{n_{C u} \sigma_{C u-2 p^{3 / 2}} T_{C u}}\left[1-k_{C u}^{C u}\right]+\frac{I_{B i}^{X P S}}{I_{C u}^{X P S}} \cdot\left[1-k_{C u}^{B i}\right]}
$$

\section{XPS parameters evaluation}

Term $n_{A}$ is estimated using the following relationship:

$$
n_{A}=\frac{\rho_{A} N_{a v o}}{M_{A}} \times d_{A}
$$

With $\rho_{A}$ being the density, $N_{a v o}$ the number of atoms per mole, $M_{A}$ the molar mass and $d_{A}$ the monolayer thickness, the followings values were obtained:

$n_{B i} \approx 9.3$ atoms $/ \mathrm{nm}^{2}$ and $n_{C u} \approx 19.2$ atoms $/ \mathrm{nm}^{2}$.

Scofield tabulation ${ }^{22}$ was used to get the cross-section $\sigma$ for the chosen transitions:

$$
\begin{aligned}
\sigma_{C u-2 p^{3 / 2}} & =15.87 \\
\sigma_{B i-4 f^{7 / 2}} & =13.85 \\
\sigma_{B i-4 f^{5 / 2}} & =10.93
\end{aligned}
$$

For $\lambda_{A}^{M}$, the same expression ${ }^{17,18}$ was used as in AES parameters evaluation and led, for $\mathrm{Cu}-2 \mathrm{p}^{3 / 2}$, to:

$$
\begin{aligned}
& \lambda_{\mathrm{Cu}}^{B i} \approx 1.051 \mathrm{~nm}(\approx 3.2 \mathrm{Bi} \text { monolayers }) \text { and } \\
& \lambda_{\mathrm{Cu}}^{\mathrm{Cu}} \approx 0.703 \mathrm{~nm}(\approx 3.1 \mathrm{Cu} \text { monolayers }) .
\end{aligned}
$$

Factor $T$ depends only on the photoelectron kinetic energy. Rather than using an empirical equation describing this dependency, we have chosen to evaluate the $T_{B i} / T_{C u}$ ratio using the pure copper spectrum obtained under the same analysis conditions on a reference sample and assuming that $T_{B i-4 f} \approx T_{C u-3 p}$. This assumption is supported by both the proximity of energy levels (1089.6 and $1094.6 \mathrm{eV}$ for Bi-4f as compared with 1176.6 for $\mathrm{Cu}-3 \mathrm{p}$ ) and the very limited evolution of $T$ for high kinetic energies. For pure copper, intensities can be written as follows:

$$
\begin{aligned}
I_{C u}^{0}\left(2 p^{3 / 2}\right) & =K^{0} N_{C u} \lambda_{C u-2 p^{3 / 2}}^{C u} \sigma_{C u-2 p^{3 / 2}} T_{C u-2 p^{3 / 2}} \\
I_{C u}^{0}(3 p) & =K^{0} N_{C u} \lambda_{C u-3 p}^{C u}\left[\sigma_{C u-3 p^{1 / 2}}+\sigma_{C u-3 p^{3 / 2}}\right] T_{C u-3 p}
\end{aligned}
$$

and therefore:

$$
\frac{T_{C u-3 p}}{T_{C u-2 p^{3 / 2}}}=\frac{I_{C u-3 p}^{0}}{I_{C u-2 p^{3 / 2}}^{0}} \times \frac{\lambda_{C u-2 p^{3 / 2}}^{C u}}{\lambda_{C u-3 p}^{C u}} \times \frac{\sigma_{C u-2 p^{3 / 2}}}{\left[\sigma_{C u-3 p^{1 / 2}}+\sigma_{C u-3 p^{3 / 2}}\right]}
$$


Using the same semiempirical equation ${ }^{17,18}$ for the IMFP, and Scofield tabulation ${ }^{22}$ for the photoionisation cross-section, the previous relationship becomes:

$$
\frac{T_{C u-3 p}}{T_{C u-2 p^{3 / 2}}}=\frac{I_{C u-3 p}^{0}}{I_{C u-2 p^{3 / 2}}^{0}} \times 2.8
$$

Experimentally, we obtained: $\frac{I_{\mathrm{C} u-3 p}^{0}}{I_{\mathrm{C} u-2 p^{3 / 2}}^{0}} \approx 0.16$, leading to:

$$
\left(\frac{T_{B i-4 f}}{T_{C u-2 p^{3 / 2}}} \approx\right) \frac{T_{C u-3 p}}{T_{C u-2 p^{3 / 2}}} \approx 0.44 .
$$

That is to be compared with the value 0.74 obtained with the help of an empirical relationship between $T$ and $E_{c}: T=2.5 \exp \left(-0.23 E_{c}\right)+1.49-5.76 \times 10^{-4} E_{c}+8.74 \times$ $10^{-8} E_{c}^{2}$, as provided by Cameca.

Using the previously described parameters calculation, the following relationship appears:

$$
\tau_{X P S} \approx \frac{\frac{I_{B i}^{X P S}}{I_{C u}^{X P S}}}{0.10+0.28 \times \frac{I_{B i}^{X P S}}{I_{C u}^{X P S}}}
$$

\section{XPS results}

Experimental intensities have been determined using area calculations with linear subtraction on direct spectra (Fig. 6). Experimental bismuth to copper intensity ratios and corresponding fractions of bismuth monolayer are presented in Table 2 .

The only parameter evaluations that can add discrepancy to the results concern the IMFP. The same empirical equation ${ }^{20}$ as proposed in AES discrepancy evaluation can be used and changes Eqn (31) into Eqn (32).

$$
\tau_{X P S} \approx \frac{\frac{I_{B i}^{X P S}}{I_{C u}^{X P S}}}{0.08+0.22 \frac{I_{B i}^{X P S}}{I_{C u}^{X P S}}}
$$

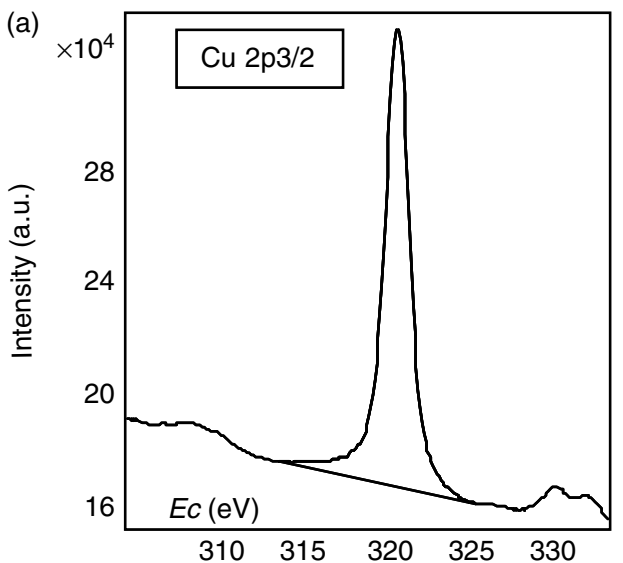

Table 2. Experimental bismuth to copper XPS intensity ratios (obtained using area evaluation on the direct XPS spectra) and their corresponding fractions of bismuth monolayer $\tau_{X P S}$ obtained using Eqn (31) for discrete summation: indicated discrepancies are those introduced by the graphical area evaluation

\begin{tabular}{lcc}
\hline & S048-1 & S048-2 \\
\hline$I_{B i}^{X P S}$ & $0.095 \pm 0.01$ & $0.115 \pm 0.01$ \\
$I_{C u}^{X P S}$ & $0.75 \pm 0.06$ & $0.87 \pm 0.06$ \\
$\tau_{X P S}$ & \\
\hline
\end{tabular}

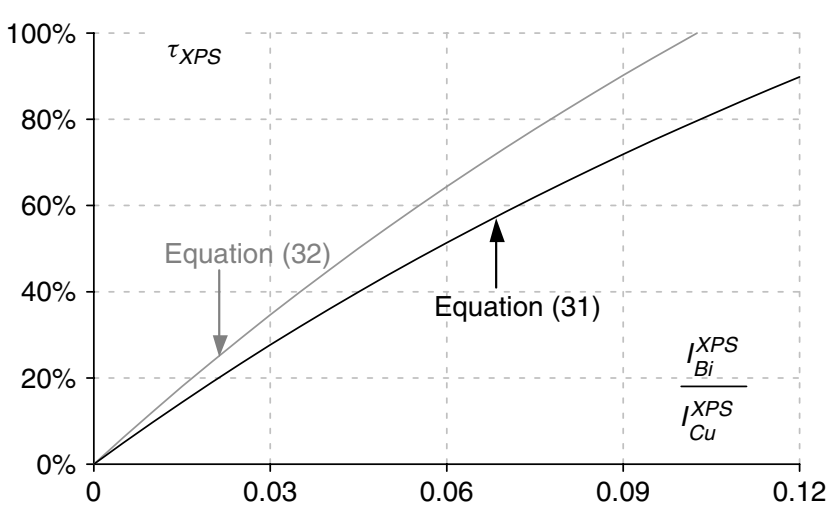

Figure 7. Graphical evolution of $\tau_{X P S}$ as a function of $\frac{l_{B i}^{X P S}}{l_{X P S}^{X P S}}$ depending on the inelastic mean free path evaluation: black curve is obtained with Eqn (31) and grey curve with Eqn (32).

It leads to significant deviations from our $\tau_{X P S}$ calculations (Fig. 7). In the case of S048-1, 0.92 monolayer of bismuth is obtained with Eqn (32) instead of 0.75 monolayer with Eqn (31). Then, it appears that XPS quantification results depend strongly on the choice of the inelastic mean free path evaluation.

\section{RBS quantification}

Referred to as a nuclear analysis technique, the RBS is based on the measurement of the scattering yield of high-energy light ions, accelerated in the MeV domain. RBS enables absolute determination of elemental concentrations, since the

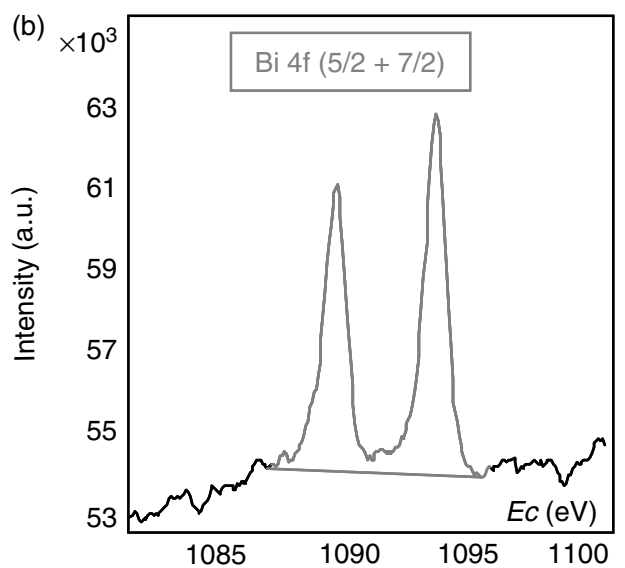

Figure 6. Direct XPS spectra from the surface for sample 1 (in situ fractured S048-1 specimen): (a) Cu2 $\mathrm{p}^{3 / 2}$ peak and (b) Bi4f $\mathrm{f}^{5 / 2}$ and $\mathrm{Bi} 4 \mathrm{f}^{7 / 2}$ peaks. 
scattering cross-sections depend only on nuclear parameters. Thus, as a first approximation, data derived from RBS spectra are independent of the chemical environment of the elements and do not require acquisitions on reference or standard samples.

\section{Data analysis}

The present experiments have been conducted with a 2.5$\mathrm{MeV}^{4} \mathrm{He}^{+}$beam and a particle detector placed at $170^{\circ}$ from the beam axis ( $c f$ Figure 8). The usual treatment of RBS data is based on simulation programs such as SIMNRA code ${ }^{23}$ by adjustment of computed spectra on experimental ones. However, in the case of ultra-thin bismuth layers on copper substrate, the spectra are simple enough to be treated manually. The foreseen advantage of a manual procedure is an easy access to the values of the physical parameters involved, which enables an evaluation of precision and accuracy.

Considering a thin sample to be analysed, the corresponding number of backscattered particles detected for a given element may be expressed as follows:

$$
I^{R B S}=\sigma(E, \Theta) \times \Omega \times Q \times N \times h
$$

with:

$\sigma(E, \Theta)$ is the scattering cross-section on the element at the energy $E$ of the incident particles (supposed to be constant within the sample) and for the scattering angle $\Theta$ (defined by the position of the particle detector),

$\Omega$ the solid angle spanned by the detector,

$Q$ the total number of incident particles,

$N$ the atomic density of the element in the sample and

$h$ the thickness of the slab.

Note that the $N \times h$ product is the number of target atoms in a unit of area and corresponds to the atomic (or mass) thickness of the slab, whatever its density. To be compared to AES and XPS results, this value will have to be converted in the bismuth monolayer fraction $\tau_{R B S}$ ( $c f$ Eqn (42) hereafter).

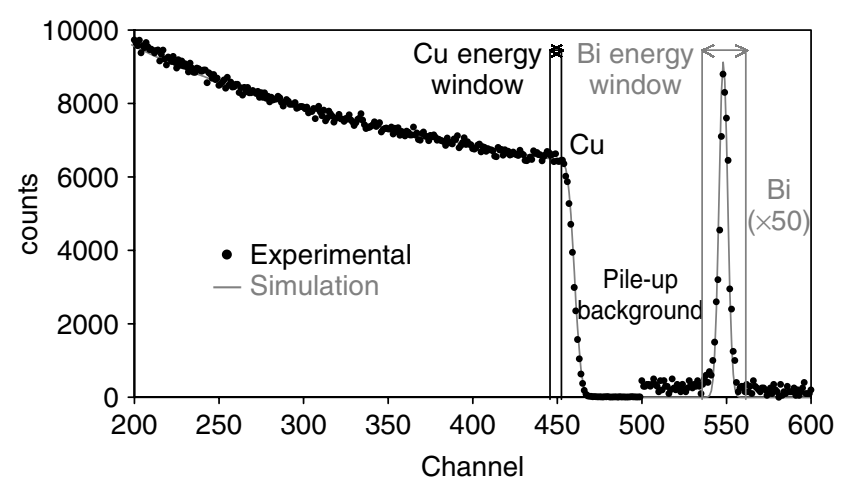

Figure 8. Experimental RBS spectrum of copper crystal surface covered with bismuth, obtained with $2.5-\mathrm{MeV} 4 \mathrm{He}^{+}$ beam and a detection angle of $170^{\circ}$. The energy $\mathrm{Bi}$ and $\mathrm{Cu}$ windows considered for quantification are indicated. $\mathrm{A}$ simulation of the data with the SIMNRA code is also plotted.
In the case of an ultra-thin bismuth layer on the top of a bulk copper sample, the bismuth appears in the spectrum as a single Gaussian peak, whereas the cooper produces the usual RBS step ( $c f$ Figure 8 ). The two signals are interference-free, except a very low smooth background under the bismuth peak due to pile-up from scattering on copper. The Eqn (33) can be directly applied to quantify the bismuth layer since the scattering occurs on the surface of the sample, exactly at the energy of the beam $(2.5 \mathrm{MeV})$. The precision and accuracy on the determined bismuth content depend on each term of Eqn (33), as discussed below.

\section{RBS parameters evaluation}

The scattering cross-sections are accurately known. Basically, their energy and angular dependence follow Rutherford's model based on Coulombian interactions:

$$
\begin{aligned}
\sigma_{\mathrm{R}}(\mathrm{E}, \Theta)= & 5.1837 \times 10^{6}\left(\frac{Z_{1} Z_{2}}{E}\right)^{2} \\
& \times \frac{\left[\left(M_{2}^{2}-M_{1}^{2} \sin ^{2} \Theta\right)^{1 / 2}+M_{2} \cos \Theta\right]^{2}}{M_{2} \sin ^{4} \Theta\left(M_{2}^{2}-M_{1}^{2} \sin \Theta\right)^{1 / 2}}
\end{aligned}
$$

where $Z_{1}$ and $M_{1}$ are the nuclear charge and the mass of the projectile, respectively, and $Z_{2}$ and $M_{2}$ those of target atom.

Experimental measurements indicate that actual crosssections depart from Rutherford's. In the present case, these deviations are taken into account, but they are low. At low energy ( $<\mathrm{a}$ few $\mathrm{MeV}$ ), a partial screening of the nuclear charges by the electron shells occurs. A correction factor $F$ has to be applied and:

$$
\sigma=F \times \sigma_{\mathrm{R}}
$$

Several semiempirical expressions have been proposed to take into account the energy and angular dependence of the correction factor ${ }^{24,25}$ but, for $\Theta>90^{\circ}$, all the models tend to the same value, depending only on the energy and on the target-projectile pair. For a $2.5-\mathrm{MeV}^{4} \mathrm{He}^{+}$beam on bismuth, the correction factor at $170^{\circ}$ is lower than $1 \%$ and can be accurately determined.

Departures from Rutherford cross-sections may also occur from the presence of short-range nuclear forces. These forces become significant only for a very short distance between the projectile and the target nuclei, i.e. either for high-energy projectiles or light target atoms (limited Coulombian repulsion between target and projectile). At $170^{\circ}$ for a $2.5-\mathrm{MeV}^{4} \mathrm{He}^{+}$beam, this deviation from Rutherford scattering no longer excess $4 \%$ for fluorine $\left(Z_{2}=9\right)$ and is insignificant for bismuth $\left(Z_{2}=83\right)$.

Another source of uncertainty is obviously the estimation of the yield of the bismuth backscattering from the bismuth peak. In our experimental conditions, the integral of the bismuth peak for a monolayer is typically 1000 counts. On the basis of a simple $\sqrt{N} / N$ estimator, the precision on the yield is in the order of $\pm 3 \%$. For ultra-thin bismuth layers, an additional error may come from pile-up background subtraction ( $c f$ Figure 8).

The last source of errors lies in the estimation of the product $\Omega \times Q$. Two methods may be applied: 
- Absolute determination of $Q$ and $\Omega$ : Since the beam intensity usually varies during the acquisition, the number of incident particles cannot be accurately deduced from beam time. A direct measurement of $Q$ is made from the charge induced in the sample by the ions of the beam (each individual ${ }^{4} \mathrm{He}^{+}$ion brings one elemental charge). Because the ion beam induces emission of secondary electrons, whose loss raises the positive charge of the sample, a $90-\mathrm{V}$ positive bias is applied to the sample holder to collect them again. The typical accuracy of charge measurements is within $3-4 \%$, sometimes higher (incomplete trapping of secondary electron produces systematic errors).

An estimation of the solid angle $\Omega$ can be set from the dimensions and the position of the detector holder, but the most accurate value should be deduced from an acquisition on a standard sample. However, as this latter measurement needs also charge measurement, the accuracy cannot be significantly better than $2 \%$.

To improve the precision, a second method, based on internal standardization described below, has been preferred.

- Use of copper as internal standard: Equation (33) can be applied only when the scattering cross-section remains constant, that is to say, in the case of a thin layer. It remains valid when considering a thin slab of the copper substrate, which contributes to the spectrum by $I_{\mathrm{Cu}}^{R B S}$. As bismuth and copper are detected simultaneously, the same $\Omega \times Q$ product applies. Then the bismuth film thickness $h_{B i}$ can be deduced from:

$$
N_{B i} \times h_{B i}=\frac{I_{B i}^{R B S}}{I_{C u}^{R B S}} \times \frac{\sigma_{C u}(E, \Theta)}{\sigma_{B i}(E, \Theta)} \times N_{C u} \times h_{C u}
$$

Like for bismuth, the scattering cross-sections on copper are practically Rutherford, with a very low screening contribution $(F)$ and no effect of short-range nuclear interactions. The ratio $\sigma_{C u}(E, \Theta) / \sigma_{B i}(E, \Theta)$ is then known accurately.

The issue for an accurate internal standardization is to link the yield of backscattering $I_{C u}^{R B S}$ to the proper quantity of copper analysed $N_{C u} \times h_{C u}$. Whereas for bismuth, the integral of the RBS peak, $I_{B i}^{R B S}$, represents the whole film, for the copper $I_{C u}^{R B S}$ is related to a slab whose thickness depends on the stopping power of ${ }^{4} \mathrm{He}$ ions in copper. This slab contributes to the content of a few channels in the spectrum, which defines an energy window of width $\delta e$ ( $c f$ Figure 8). $\delta e$ comes from the longer path of the particles backscattered at the bottom of the slab since they have to go twice through the layer, before and after the backscattering event.

Introducing $[\varepsilon]$, the stopping cross-section factor of the particles of the beam, the depth scale is given by:

$$
\delta e=[\varepsilon] \times N_{C u} \times h_{C u}
$$

which gives, combined with Eqn (34):

$$
N_{B i} \times h_{B i}=\frac{I_{B i}^{R B S}}{I_{C u}^{R B S}} \times \frac{\sigma_{C u}(E, \Theta)}{\sigma_{B i}(E, \Theta)} \times \frac{\delta e}{[\varepsilon]}
$$

The energy loss of the particles of the beam in the sample varies with their energy. Then, $[\varepsilon]$ is not a primary parameter since it combines both inward and outward paths, before and after the scattering, respectively. It can be computed in any case, but the simplest expression comes in the case of a surface slab, when:

$$
\delta e=K \times E_{0}-E_{1}
$$

where $E_{0}$ is the energy of the particles of the beam, $K$ the kinematic factor and $E_{1}$ the energy of the detected particles coming from the depth $h_{C u}$. It comes from Eqns (35) and (37):

$$
[\varepsilon]=\left(K \times \varepsilon_{\text {in }}+\frac{1}{\cos \theta} \times \varepsilon_{\text {out }}\right)
$$

with $\theta$ being the angle between the beam and the backscattered particles (with the sample normal to the beam). $\varepsilon_{\text {in }}$ and $\varepsilon_{\text {out }}$ are the stopping cross sections along the inward and outward paths, practically constant for a thin layer. The values of $\varepsilon_{\text {in }}$ and $\varepsilon_{\text {out }}$ are taken for energies $E_{0}$ and $K E_{0}$, respectively.

\section{RBS results}

The example of quantification given below is based on the spectrum of Fig. 8 from which values of $I_{B i}^{R B S}, I_{\mathrm{Cu}}^{R B S}$ and $\delta e$ are directly extracted. $I_{B i}^{R B S}$ is the net area of the bismuth peak (1158 counts), pile-up background deducted. $I_{\mathrm{Cu}}^{R B S}$ is the content of an energy window open in the surface region of the copper step (32341 counts integrated in five channels). $\delta e$ is the energy width of this window deduced from the energy calibration of the particle detector $(5 \times 4.22 \mathrm{keV} /$ channel).

The geometry of detection defines a scattering mean angle $\Theta$ of $170^{\circ}$. At the energy of the incident ${ }^{4} \mathrm{He}$ beam $(2.5 \mathrm{MeV})$, the corresponding cross-sections $\sigma_{C u}(2.5,170)$ and $\sigma_{B i}(2.5,170)$ are $700 \mathrm{mb} / \mathrm{sr}$ and $5712 \mathrm{mb} / \mathrm{sr}$, respectively. The kinematic factor $K$ for ${ }^{4} \mathrm{He}^{+}$scattering on copper is equal to 0.778 at $170^{\circ}$ and the angle $\theta$ is $10^{\circ}$.

The stopping cross-sections of light ions are accurately known for medium and heavy target elements. Although an overall increase of $\varepsilon$ on the mass of the target element may be easily computed, departures from the mean law exist. A decrease in the stopping cross section occurs when $d$-shell electrons are added in the sequence of transition elements, such as from $\mathrm{Ca}$ to $\mathrm{Cu}$ or from $\mathrm{Nb}$ to $\mathrm{Ag}$. In this case, the electron density near the atom increases enough to reduce the average electron density seen by an energetic particle traversing the material. The copper corresponds to a minimum with $\varepsilon$ values lowered by typically $10 \%$ below a mean law value.

To benefit from accurate values, stopping cross-sections in copper have been computed with the Ziegler's SRIM2003 code. ${ }^{26,27} \varepsilon_{\text {in }}$ is found to be $61.5 \mathrm{eV} / 10^{15}$ at $\mathrm{cm}^{-2}$ for $2.5 \mathrm{MeV}$ ${ }^{4} \mathrm{He}$ and $\varepsilon_{\text {out }}$ to be $66.6 \mathrm{eV} / 10^{15}$ at $\mathrm{cm}^{-2}$ for $1.946 \mathrm{MeV}{ }^{4} \mathrm{He}$ $\left(K E_{0}\right)$.

From the injection of these values in Eqn (36), it becomes:

$$
N_{B i} \times h_{B i}=0.81 \times 10^{15} \text { at } \mathrm{cm}^{-2}
$$

The bismuth monolayer fraction $\tau_{R B S}$ can be related to this value with the help of the atomic thickness of a bismuth monolayer $n_{B i}$ :

$$
N_{B i} \times h_{B i}=\tau_{R B S} \times n_{B i}
$$


Taking into account the evaluation of the thickness of a monolayer presented in the AES section (cube root of the atomic volume), $n_{B i}$ is evaluated as $0.93 \times 10^{15}$ at $\mathrm{cm}^{-2}(=9.3$ at $\mathrm{nm}^{-2}$ ). The thickness of the bismuth layer of Fig. 8 is then estimated to be:

$$
\tau_{R B S}=\frac{N_{B i} \times h_{B i}}{n_{B i}} \approx 0.87 \text { monolayer }
$$

The results for two samples, S048-1 and S137-1 are reported in Table 3.

The uncertainties on these thickness may be easily estimated from Eqn (36). Errors on $I_{B i}^{R B S}$ and $I_{C u}^{R B S}$ are only statistical fluctuations. Taking the square root of the value as an estimator, typical errors are $\pm 0.5 \%$ for $I_{C u}^{R B S}$ and $\pm 3 \%$ for $I_{B i}^{R B S}$. The cross-sections $\sigma_{C u}(2.5,170)$ and $\sigma_{B i}(2.5,170)$ are accurately known, better than $\pm 1 \%$, since the correction factor from Rutherford cross-sections due to screening effects is very close to 1 in our experimental conditions. The accuracy of $\delta e$ depends on the quality of the energy calibration of the particle detector, typically $\pm 0.5 \%$ provided a broad range of energy positions of RBS steps have been checked (target elements of different mass).

The main source of inaccuracy is the estimation of the stopping cross-sections. Ziegler's SRIM code calculations are based on a quantum mechanical treatment of ion-atom collisions, validated on experimental data. ${ }^{1} \mathrm{H}$ and ${ }^{4} \mathrm{He}$, as projectiles, have been by far the most studied, and the corresponding models for their respective stopping crosssections produce values with a good accuracy, below $4 \%$ in our present energy range.

In conclusion, the relative precision of RBS bismuth thickness measurements depends on statistical fluctuations on the bismuth and copper signals, typically $\pm 3.5 \%$, whereas the accuracy is dominated by stopping-power estimation $\pm 4 \%$. The overall accuracy may be then estimated to $\pm 10 \%$.

Table 3. Experimental fractions of bismuth monolayer $\tau_{R B S}$ obtained by RBS analyses

\begin{tabular}{ccc}
\hline & S048-1 & S137-1 \\
\hline$\tau_{\text {RBS }}$ & $0.87 \pm 0.08$ & $0.87 \pm 0.08$ \\
\hline
\end{tabular}

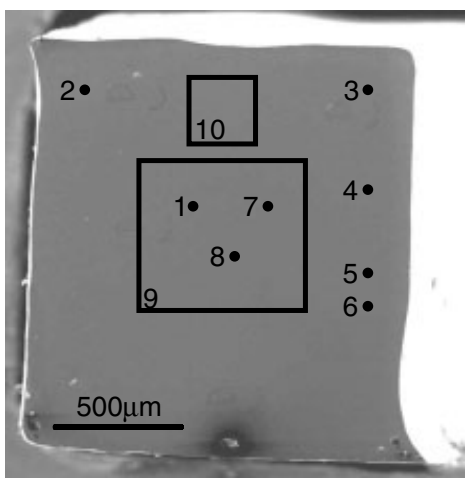

\begin{tabular}{|c|c|c|c|}
\hline Position & $\tau_{\text {AES }}$ & Position & $\tau_{\text {AES }}$ \\
\hline 1 & 0.78 & 6 & 0.81 \\
\hline 2 & 0.82 & 7 & 0.83 \\
\hline 3 & 0.76 & 8 & 0.81 \\
\hline 4 & 0.78 & 9 & 0.85 \\
\hline 5 & 0.82 & 10 & 0.84 \\
\hline
\end{tabular}

Figure 9. SEM picture of the S048-1 fracture surface: points are AES spot analyses (1-8) whereas squares are AES scan analyses $(9-10)$. Bismuth coverage is given (in fraction of monolayer) for each analysis according to the procedure described in a previous paragraph and using Eqn (17): the average $\tau_{\text {AES }}$ value is 0.81 for this example.

\section{DISCUSSION}

\section{Validation of physical assumptions}

The purpose stated in the introduction was to perform comparative measurements using complementary methods (XPS, AES and RBS) in order to get a reliable picture of the quantity of bismuth present in a copper grain boundary after a contact between the solid copper bicrystal and liquid bismuth. To achieve this goal, two main assumptions had to be stated, namely: homogeneous bismuth distribution on the fracture surfaces and equirepartition.

The first assumption that a homogeneous layer of bismuth is present on the copper substrate is critical for XPS because of a very large $(3 \mathrm{~mm})$ spot size of a twin anode used. Figure 9 shows a series of AES analyses made on different locations of a fracture surface. It clearly indicates that bismuth coverage is almost constant on the whole specimen surface, confirming our assumption.

The second assumption concerns the equirepartition of bismuth after the fracture: it is needed to get valuable information about the initial intergranular bismuth presence before the fracture. Figure 10 shows two RBS analyses made on two opposite sides of a fracture surface. It confirms that bismuth is equally distributed on both sides of the fracture surface. This is in agreement with a previous study based on AES analyses made on copper-bismuth alloys fracture surfaces where the bismuth concentration was similar on the two matching fracture surfaces. ${ }^{28}$

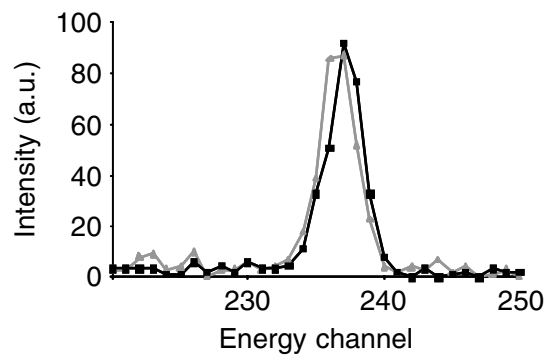

Figure 10. RBS experimental bismuth peaks obtained on two sides of the fracture surface of the same sample: the same quantity of bismuth is present on both of them (intensities have been normalised with respect to copper signal in order to get a relevant comparison). 


\section{Validation of the mathematical models}

Instead of using methods based on a discrete summation for AES and XPS quantifications, continuous summation can also be made. Concerning AES, this does not lead to any changes in the results, as the final equations relating bismuth-to-copper experimental intensity ratio to bismuth coverage are strictly the same (as shown in Appendix). On another hand, XPS results can vary with the choice of the summation method. With the discrete summation, Eqns (23) and (31) have been obtained. On the other hand, a continuous summation can also be used. Equations become:

$$
\begin{aligned}
I_{B i}^{X P S} & =\tau_{X P S} K_{X P S} N_{B i} \sigma_{B i} T_{B i} \times \int_{0}^{d_{B i}} \exp \left(\frac{-z}{\lambda_{B i}^{B i} \cos \theta}\right) \cdot \mathrm{d} z \\
& =\tau_{X P S} K_{X P S} N_{B i} \sigma_{B i} T_{B i}\left[1-k_{B i}^{B i}\right] \lambda_{B i}^{B i} \cos \theta
\end{aligned}
$$

and

$$
\begin{aligned}
I_{C u}^{X P S}= & K_{X P S} N_{C u} \sigma_{C u} T_{C u}\left[\left(1-\tau_{X P S}\right)+\tau_{X P S} k_{C u}^{B i}\right] \\
& \times \int_{0}^{+\infty} \exp \left(\frac{-z}{\lambda_{C u}^{C u} \cos \theta}\right) \cdot \mathrm{d} z \\
= & K_{X P S} N_{C u} \sigma_{C u} T_{C u}\left[\left(1-\tau_{X P S}\right)+\tau_{X P S} k_{C u}^{B i}\right] \lambda_{C u}^{C u} \cos \theta
\end{aligned}
$$

Term $N$ is the number of atoms per unit volume estimated using the following relationship: $N=\frac{\rho \times N_{\text {avogadro }}}{M}$. The followings values were used:

$$
N_{B i} \approx 2.82 \times 10^{28} \text { atoms } \mathrm{m}^{-3} \text { and } N_{C u} \approx 8.43 \times 10^{28} \text { atoms }
$$
$\mathrm{m}^{-3}$.

The inelastic mean free path $\lambda_{B i}^{B i}$ was then calculated:

$\lambda_{B i}^{B i} \approx 2.57 \mathrm{~nm}$ (7.8 monolayers) for both Bi $4 \mathrm{f}^{5 / 2}$ and $4 \mathrm{f}^{7 / 2}$ kinetic energies

In this case, the fraction of bismuth monolayer $\tau_{X P S}$ present on the fracture sample can be related to the experimental bismuth-to-copper ratio with the following equation:

$$
\tau_{X P S}=\frac{\frac{I_{B i}^{X P S}}{I_{C u}^{X P S}}}{\frac{N_{B i} \lambda_{B i}^{B i}\left[\sigma_{B i-4 f^{7 / 2}}+\sigma_{B i-4 f^{5 / 2}}\right] T_{B i}}{N_{C u} \lambda_{C u}^{C u} \sigma_{C u-2 p^{3 / 2}} T_{C u}}\left[1-k_{B i}^{B i}\right]+\frac{I_{B i}^{X P S}}{I_{C u}^{X P S}}\left[1-k_{C u}^{B i}\right]}
$$

Using the previously described parameters calculation, the following relationship appears:

$$
\tau_{X P S} \approx \frac{\frac{I_{B i}^{X P S}}{I_{C u}^{X P S}}}{0.09+0.28 \times \frac{I_{B i}^{X P S}}{I_{C u}^{X P S}}}
$$

Equation (43) is rather similar to Eqn (31) and that proves the equivalence between the two approaches: both discrete and continuous summations give consistent results with each others.

\section{Inelastic mean free paths versus effective attenuation lengths}

The reasons that led us to use IMFP instead of effective attenuation lengths ${ }^{29}$ (EAL) in both AES and XPS quantifications are the following:
- we wanted to get a direct comparison between our studies made on the copper/bismuth system and previous studies made with equivalent procedures on the nickel/bismuth system where the authors had used the IMFP in their AES quantification, ${ }^{9}$

- taking IMFP instead of EAL in the quantifications leads clearly to an overestimation of the bismuth coverage as the EAL are usually about 10 to $20 \%$ less than the IMFP; nevertheless, this 'intentional' overestimation makes us feel even more confident on our conclusions about the absence of grain-boundary wetting (see the following text).

\section{Coherency of the results}

AES measurements have shown the presence of $82 \pm 18 \%$ of bismuth monolayer on the analysed fracture surfaces. On another hand, XPS measurements have shown the presence of $81 \pm 12 \%$ of bismuth monolayer on the same surfaces, the uncertainty being due to the spectra exploitation rather than to the physical parameters calculations. RBS measurements have shown the presence of $87 \pm 8 \%$ of bismuth monolayer on the same surfaces. As a conclusion, all results are therefore consistent with each other, with a significantly larger deviation for AES measurements.

Our results are very close to those obtained by AES quantification of bismuth grain boundary segregation into copper. ${ }^{30-32}$ As a matter of fact, our study shows that the contact between solid bicrystalline copper and liquid bismuth leads to the presence of about $2 \times 0.8=1.6$ monolayers of bismuth in the copper grain boundary. This is in fair agreement with the upper value of two monolayers found after grain boundary segregation. So, this would indicate that even with a contact between solid copper and liquid bismuth (saturated with copper), bismuth intergranular presence is controlled by the same thermodynamic equilibrium as that of intergranular segregation: namely, the number of sites of the grain boundary energetically in favour of bismuth presence.

\section{Implications on LME phenomenon}

These results mean also that we are far from a liquid bismuth invasion of the copper grain boundary. Such a conclusion is very important as a number of models proposed to describe LME are based on the grain boundary wetting that leads to the replacement of the initial grain boundary by a nanometer-thick intergranular liquid phase. In our case, $\mathrm{Cu} / \mathrm{Bi}$ system after heat-treatment at $500^{\circ} \mathrm{C}$ for 137 hours, we did not observe such a phenomenon. This is in apparent contradiction with earlier results obtained using XPS for the same $\mathrm{Cu} / \mathrm{Bi}$ system where several monolayers of bismuth were thought to exist in a copper grain boundary ${ }^{6}$ (heat treatment: $8 \mathrm{~h}$ at $600^{\circ} \mathrm{C}$ ). Yet, Joseph ${ }^{33}$ made complementary AES analyses on a $2.0 \times 3.5 \mathrm{~mm}^{2}$ bicrystalline fracture surface (heat treatment: $2 \mathrm{~h}$ at $600{ }^{\circ} \mathrm{C}$ ) and found an homogeneous bismuth coverage of about $93 \%$ of the monolayer, which is in reasonable agreement with our present results.

This work strongly suggests that intergranular diffusion can be the mechanism responsible for bismuth grain boundary penetration into copper. Additional tests have 
been performed in order to get information on the kinetics of this penetration. As this kinetics appears to be parabolic, ${ }^{15,34}$ it strengthens the idea that intergranular diffusion of bismuth in copper is the mechanism controlling the liquid-bismuth induced intergranular embrittlement of copper.

\section{CONCLUSIONS}

Intergranular embrittlement of bicrystalline copper by liquid bismuth has been achieved at $500^{\circ} \mathrm{C}$ using condensation of bismuth vapour on copper in sealed silica tubes without any applied stress (durations: 48 and $137 \mathrm{~h}$ ). The resulting room temperature brittleness allowed in situ fractures for XPS and AES analyses as well as further $e x$ situ RBS analyses.

AES, XPS and RBS measurements are in excellent agreement, showing the presence of about 1.6 to 1.7 monolayers of bismuth in the copper grain boundary: $2 \times 0.82 \approx 1.6$ monolayers according to AES analyses, $2 \times 0.81 \approx 1.6$ monolayers according to XPS analyses and $2 \times 0.87 \approx 1.7$ monolayers according to RBS analyses.

The quantification models were based on two assumptions that were both verified in this study. AES quantification allowed to validate the assumption of a homogeneous repartition of bismuth. On another hand, the equirepartition of bismuth into both sides of the fracture surfaces was verified using RBS measurements.

These results clearly indicate that the contact between copper bicrystal and liquid bismuth does not produce any grain-boundary wetting at $500{ }^{\circ} \mathrm{C}$. As a matter of fact, the bismuth coverages obtained in this study are very close to those obtained in the same system for grain boundary segregation.

\section{Acknowledgements}

The authors wish to thank G. Santarini and A. Terlain (both from the French Atomic energy Commission) for fruitful discussions as well as the crew of the nuclear microprobe of the Laboratoire Pierre SUE, especially L. Daudin, H. Khodja and J.P. Gallien.

\section{APPENDIX}

When trying to quantify bismuth presence on a copper substrate, two different approaches can be used. Both introduce the assumption that bismuth coverage is present as a fraction of monolayer $\tau_{A E S}$. First, using a discrete summation (monolayer by monolayer), the AES bismuth to copper intensity ratio can be related to $\tau_{A E S}$ with:

$$
\frac{I_{B i}^{A E S}}{I_{C u}^{A E S}}=\frac{I_{B i}^{0} R_{B i}^{C u}}{I_{C u}^{0} R_{B i}^{B i}} \frac{1-k_{p}^{B i} k_{B i}^{B i}}{\frac{\left(1-\tau_{A E S}\right)}{\tau_{A E S}}+k_{p}^{B i} k_{C u}^{B i}}
$$

(see the body of the article for more details)

Second, using a continuous summation, with the same notations presented for the previous approach and introducing $N$ : number of atoms per unit volume and $\alpha$ (parameter that includes, like $\beta$, the ionisation cross section, the deexcitation probability and the analyser transmission function), the following equations are obtained:

$I_{B i}^{A E S}=\tau_{A E S} K_{A} \alpha_{B i} N_{B i} R_{B i}^{C u} \int_{0}^{d_{B i}} \exp \left(\frac{-z}{\lambda_{p}^{B i} \cos \varphi}\right)$

$$
\begin{aligned}
& \times \exp \left(\frac{-z}{\lambda_{B i}^{B i} \cos \theta}\right) \cdot \mathrm{d} z \\
= & \tau_{A E S} K_{A} \alpha_{B i} N_{B i} R_{B i}^{C u} \frac{\left[1-k_{p}^{B i} k_{B i}^{B i}\right]}{\left[\frac{1}{\lambda_{p}^{B i} \cos \varphi}+\frac{1}{\lambda_{B i}^{B i} \cos \theta}\right]} \\
I_{C u}^{A E S}= & K_{A} \alpha_{C u} N_{C u} R_{C u}^{C u}\left[\tau_{A E S} k_{p}^{B i} k_{C u}^{B i}+\left(1-\tau_{A E S}\right)\right] \\
& \times \int_{0}^{+\infty} \exp \left(\frac{-z}{\lambda_{p}^{C u} \cos \varphi}\right) \exp \left(\frac{-z}{\lambda_{C u}^{C u} \cos \theta}\right) \cdot d z \\
= & \left.\tau_{A E S} K_{A} \alpha_{C u} N_{C u} R_{C u}^{C u} \frac{\left[\frac{\left(1-\tau_{A E S}\right)}{\left[\frac{1}{\tau_{A E S}}+k_{p}^{B i} k_{C u}^{B i}\right.}\right]}{\lambda_{p}^{C u} \cos \varphi}+\frac{1}{\lambda_{C u}^{C u} \cos \theta}\right] \\
= & \frac{K_{A}^{0} \alpha_{B i} N_{B i} R_{B i}^{B i} \int_{0}^{+\infty} \exp \left(\frac{-z}{\lambda_{p}^{B i} \cos \varphi}\right) \exp \left(\frac{-z}{\lambda_{B i}^{B i} \cos \theta}\right) \cdot \mathrm{d} z}{\left[\frac{1}{\lambda_{p}^{B i} \cos \varphi}+\frac{1}{\lambda_{B i}^{B i} \cos \theta}\right]} \\
&
\end{aligned}
$$

and

$$
\begin{aligned}
I_{C u}^{0} & =K_{A}^{0} \alpha_{C u} N_{C u} R_{C u}^{C u} \int_{0}^{+\infty} \exp \left(\frac{-z}{\lambda_{p}^{C u} \cos \varphi}\right) \exp \left(\frac{-z}{\lambda_{C u}^{C u} \cos \theta}\right) \mathrm{d} z \\
& =\frac{K_{A}^{0} \alpha_{C u} N_{C u} R_{C u}^{C u}}{\left[\frac{1}{\lambda_{p}^{C u} \cos \varphi}+\frac{1}{\lambda_{C u}^{C u} \cos \theta}\right]}
\end{aligned}
$$

and thus:

$$
\frac{I_{B i}^{A E S}}{I_{C u}^{A E S}}=\frac{I_{B i}^{0} R_{B i}^{C u}}{I_{C u}^{0} R_{B i}^{B i}} \frac{1-k_{p}^{B i} k_{B i}^{B i}}{\frac{\left(1-\tau_{A E S}\right)}{\tau_{A E S}}+k_{p}^{B i} k_{C u}^{B i}}
$$

that is, again, Eqn (48).

It can appear useful to relate together the two parameters $\alpha$ and $\beta$. Comparing the equations obtained for pure elements according to both the discrete and the continuous summations and assuming $n_{A}=d_{A} \times N_{A}$, it comes: $\alpha_{A}=$ $\beta_{A}\left[\frac{d_{A}}{\lambda_{p}^{A} \cos \varphi}+\frac{d_{A}}{\lambda_{A}^{A} \cos \theta}\right]$ $1-k_{p}^{A} k_{A}^{A}$ decreasing to $0, \alpha_{A}=\beta_{A}$.

\section{REFERENCES}

1. Bauer GS, Salvatores M, Heusener G. J. Nucl. Mater. 2001; 296: 17.

2. Bauer GS, Dai Y, Maloy S, Mansur LK, Ullmaier H. J. Nucl. Mater. 2001; 296: 321.

3. Kikuchi K, Sasa T, Mukugi K, Kai T, Ouchi N, Ioka I. J. Nucl. Mater. 2001; 296: 34.

4. Fernandes PJL, Jones DRH. Int. Mater. Rev. 1997; 42: 251.

5. Joseph B, Barbier F, Aucouturier M. Eur. Phys. J-Appl. Phys. 1999; 5: 19.

6. Joseph B, Barbier F, Dagoury G, Aucouturier M. Scripta Mater. 1998; 39: 775.

7. Joseph B, Barbier F, Aucouturier M. Mater. Sci. Forum. 1999; 735: 294.

8. Marié N, Wolski K, Biscondi M. J. Nucl. Mater. 2001; 296: 282.

9. Wolski K, Marié N, Biscondi M. Surf. Interface Anal. 2001; 31: 280. 
10. Joseph B, Barbier F, Aucouturier M. J. Phys. Iv 1999; 9: 235.

11. Fraczkiewicz A, Biscondi M. J. Phys. 1985; 46: 497.

12. Fraczkiewicz A. Ségrégation d'équilibre du bismuth aux joints de flexion pure dans le cuivre: étude expérimentale et simulation par ordinateur, Ecole des Mines de Saint-Etienne, 1986.

13. Marié N. Etude de la pénétration intergranulaire fragilisante du bismuth liquide dans le nickel solide à $700^{\circ} \mathrm{C}$, Université Jean Monnet de Saint-Etienne: Saint-Etienne (France), 2003.

14. Chang LS. Untersuchungen zur Thermodynamik und Kinetic der Korngrenzensegregation von $\mathrm{Bi}$ in $\mathrm{Cu}$. Universität Stuttgart: Stuttgart (Germany), 1998.

15. Laporte V, Wolski K. to be submitted to Scripta Materialia.

16. Shimizu R. Jpn. J. Appl. Phys. 1983; 22: 1631.

17. Tanuma S, Powell CJ, Penn DR. Surf. Interface Anal. 1991; 17: 911.

18. Tanuma S, Powell CJ, Penn DR. Surf. Interface Anal. 1993; 20: 77.

19. Tanuma S, Powell CJ, Penn DR. Surf. Interface Anal. 1994; 21: 165.

20. Seah MP. Surf. Interface Anal. 1990; 1: 201.

21. Jablonski A. Surf. Sci. 2002; 499: 19.

22. Grimblot J. L'analyse de Surface des Solides par Spectroscopies Électroniques et Ioniques. Masson: Paris, 1995.

23. Mayer M. SIMNRA User's Guide. Max-Planck-Institüt für Plasmaphysic: Garching (Germany), 1997.
24. L'Ecuyer J, Davies JA, Matsunami N. Nucl. Instrum. Methods Phys. Res. Sect. B-Beam Interact. Mater. Atoms 1979; 160: 337.

25. Andersen HH, Besenbacher F, Loftager P, Möller W. Phys. Rev. A 1980; 21: 1891.

26. Ziegler JF, Biersack JP, Littmark U. The Stopping and Range of Ions in Solids, vol. 1. Pergamon Press: New-York, 1984.

27. Ziegler JF. www.srim.org. 2004.

28. Powell BD, Mykura H. Acta Metall. 1973; 21: 1151.

29. Powell CJ, Jablonski A. Surf. Interface Anal. 2002; 33: 211.

30. Chang LS, Rabkin E, Straumal BB, Baretzky B, Gust W. Acta Mater. 1999; 47: 4041.

31. Chang LS, Rabkin E, Straumal BB, Lejcek P, Hofmann S, Gust W. Scripta Mater. 1997; 37: 729.

32. Chang LS, Rabkin E, Hofmann S, Gust W. Acta Mater. 1999; 47: 2951.

33. Joseph B. Fragilisation du Cuivre Par le Bismuth Liquide: Étude Cinétique et Mécanisme. Université de Paris XI: Orsay, 1998.

34. Laporte V, Wolski K, Berger P, Terlain A, Santarini G. Defect Diffus. Forum 2005; 683: 237. 